\title{
Experimental Evaluation of Some Exploration Strategies for Mobile Robots
}

\author{
Francesco Amigoni
}

\begin{abstract}
The ability of building maps of environments where they operate is one of the main requirements for autonomous mobile robots. An efficient map building process is based on a good exploration strategy that determines the most convenient observation positions in a partially known environment in order to incrementally map it. Several exploration strategies have been proposed in literature but their comparative evaluations are rare. In this paper, we experimentally compare some exploration strategies in order to contribute to assess their strengths and weaknesses.
\end{abstract}

\section{INTRODUCTION}

The ability to build spatial models, or maps, of environments where they operate is undoubtedly one of the main requirements for autonomous mobile robots [1]. To be efficient in map building, an autonomous robot needs a good exploration strategy that determines the most convenient observation positions in a partially known environment in order to incrementally map it. More precisely, the process of exploring an unknown environment using a mobile robot equipped with a suitable sensor can be sketched as follows:

(a) build a local map $S^{t}$ that represents the portion of the environment surrounding the robot at position $p^{t}$ ( $t$ is the current discrete time step),

(b) update the global map $M^{t}$ according to the newly acquired $S^{t}$, obtaining $M^{t+1}$, and

(c) use the exploration strategy to determine the next observation position $p^{t+1}$, reach it, and start again from (a).

Several exploration strategies have been proposed in the literature. However, only sometimes they are evaluated against other strategies (see, for example, [2], [3], [4], [5]). To the best of our knowledge, [6] is the only work that has been explicitly devoted to a comparative experimental assessment of different exploration strategies. However, the strategies compared in [6] are very primitive when compared to those proposed during the following decade. In this paper we evaluate some recent exploration strategies to provide an original contribution that, in this sense, expands and updates that of [6].

More precisely, in this paper, we experimentally compare a number of exploration strategies that represent a significant sample of those presented in literature, covering a spectrum from simple strategies that randomly move the robot to sophisticated strategies that evaluate the worthiness of possible observation positions. The comparison has been performed

\footnotetext{
F. Amigoni is with the Dipartimento di Elettronica e Informazione, Politecnico di Milano, Milano, Italy.
} francesco.amigoni@polimi.it. in simulation (as it is usual when systematic experiments are performed [3]) in different environments and with different parameters, in order to get a comprehensive assessment of the strengths and weaknesses of the approaches.

We explicitly note that the original contribution of this paper is not in proposing new exploration strategies but in experimentally analyzing some of the proposed strategies to get some insights for helping the future development of better exploration strategies.

This paper is structured as follows. The next section reviews some significant exploration strategies proposed in literature. Section III describes our experimental setting and the strategies we selected for comparison. Section IV discusses the experimental results we obtained. Section V concludes the paper.

\section{A REVIEW OF EXPLORATION STRATEGIES}

Mapping is an incremental process. Since the ranges of the sensors are limited, measurements are inaccurate, and occlusions may occur, map building is usually performed by taking several measurements of environments at different positions and by integrating these measurements in a global map. Maps are thus built incrementally by integrating measurements on the basis of the (probabilistic) estimated positions of the robot [7] or of the geometrical features of the maps [8], [9].

At a given time of the incremental process of map building, exploration strategies drive the selection of the successive observation positions, on the basis of the global map built until that time. Excluding the cases in which the robots are manually driven to acquire the measurements used to build the map, in the following we review a number of significant exploration strategies that have been presented in literature, roughly going from simple to complex approaches. We mainly concentrate on systems developed for single robots (a good survey of exploration strategies for multirobot systems is provided by [3]). We also note that not all the exploration strategies reported below are directly comparable, since they refer to different map representations (e.g., grid- or point-based). However, our aim is to provide a review of the basic ideas on which the different exploration strategies are built, abstracting from many details.

Some systems employ a fixed path to explore an environment. For example, the exploration strategies proposed in [10] include the Concentric strategy, in which a robot successively traces concentric circles starting from its initial position. [11] extends this approach using a parameterized 
trajectory to direct the exploration of the robot in an environment. Other exploration strategies make a robot to move randomly to observation positions without explicitly evaluating their worthiness [12], [13].

The most complex exploration strategies try to determine the best observation positions to improve the efficiency of exploration process. In particular, they aim at reducing the exploration time, by making a small number of exploration steps and limiting the travelled path, while building an accurate map. In general, these methods employ a greedy approach [14], that consists in moving the robot from its current location towards the next best observation position. It has been shown [15] that planning long exploration paths with only partial knowledge of the environment can result in a waste of resources. The idea on which these strategies are based is that a number of candidate observation positions are first generated and then evaluated using an evaluation function $f(\cdot)$ in order to pick out the next best observation position among the candidate ones. Some exploration strategies include in $f(\cdot)$ only the cost of reaching a candidate observation position. For example, the frontierbased exploration strategy of [16] directs a robot towards the nearest frontier, namely towards the nearest boundary between free and unknown space. Some other approaches, like that in [17], include in $f(\cdot)$ an evaluation of the utility of reaching a candidate observation position $p$, that is measured as the amount of new information about the environment that a robot is expected to gain from $p$.

One of the first exploration strategies using a combination of utility and cost for evaluating candidate observation positions is that proposed in [18]. The approach employed in [3] coordinates the exploration activities of multiple robots in order to minimize the overall exploration time. In this case, grid maps are employed and the cell in which a robot should move next is the one that maximizes the difference between a (probabilistic-based) measure of expected utility and the path cost (multiplied by a factor $\beta$ that determines the relative importance of utility versus cost). A mechanism to reduce the utility of a cell when other robots can be around is implemented. [19] describes an exploration strategy for building segment-based maps. Candidate observation positions are generated across the edge of the explored regions, in which the robot is guaranteed to move without collision risks. Candidate observation positions are evaluated using a combination of cost and utility. The exploration strategy proposed in [20] adds to cost and utility also a component relative to localizability in defining $f(\cdot)$. Localizability accounts for the expected precision of robot localization in a candidate observation position. While in [20] these three components (cost, utility, and localizability) are simply summed up, in [21] they are combined in a theoretically founded informationbased criterion. Also other exploration strategies make use of information theory to define criteria for selecting the next best observation position, for example [4], [5], [22]. In particular, [5] combines cost and utility in an entropy-based framework for finding the next best observation position.

In [15], the problem of planning optimal exploration paths is considered. Candidate observation positions along a path are evaluated with a complex evaluation function that accounts for a number of issues, including: (i) recognition of landmarks for localization, (ii) extraction of features that can be used to integrate local maps (i.e., corners), (iii) maximizing the view of unexplored areas, (iv) smoothing the robot's trajectory, and (v) minimizing the distance travelled by the robot. Finally, there have been also some attempts to consider the determination of the next best observation position as a multi-objective optimization problem [2], in which objective functions are related to cost, utility, and localizability and in which the best observation position is picked up among those on the Pareto frontier by using the concept of distance from ideal solution.

\section{THE EXPERIMENTAL SETTING AND THE SELECTED STRATEGIES}

The main contribution of this paper is to provide a comparative experimental evaluation of some exploration strategies. We compared these strategies in a common setting that is described in the following.

We assume to have an holonomic mobile robot that can move in a two-dimensional environment and that is equipped with a sensor able to perceive obstacles in the surrounding environment. We assume that the field of view of the sensor has a width of $360^{\circ}$ and a range $r$. The portion of the environment scanned by the robot during a sensing operation is thus a circle with radius $r$. In our experimental activity, we let $r$ assume different values. To be concrete, in the following we assume that this sensor represents a laser range scanner, but also some vision systems can take this form. For many purposes the simulated robot can be thought as dimensionless; we consider its size in the path planning and collision checking algorithms.

We store the map in two lists of line segments, that represent the obstacles and the boundary of the unexplored area, respectively. The first list, called obstacle list, stores the line segments representing the edges of the observed obstacles; while the second list, called free edge list, stores the line segments representing the free edges of the map, namely the boundaries between explored and unexplored areas. (Similar data structures are used, for example, in [19].) The map is updated once new sensorial data are acquired by the robot. Raw sensorial data are the points returned by the simulated sensor. We approximate them with line segments using the algorithm of [19]. The two lists of line segments are updated by inserting the new line segments just acquired. During this operation, line segments that are collinear and consecutive are fused together to reduce the dimensions of the lists. For efficiency reasons, we keep also a list of raw points perceived by the simulated sensor and a polygon representing the known area of the environment.

We used the following general algorithm for evaluating the exploration strategies.

- Generate a set of random candidate observation positions along the line segments of the free edge list, 
namely along the boundary between known and unknown space. We generate a candidate observation position for each line segment in the free edge list longer than $1.5 \mathrm{~m}$. Reacheability of a candidate observation position $p$ is checked by building a path, composed of a sequence of line segments, from the current position of the robot to $p$. Candidate observation positions that cannot be safely reached from the current position of the robot (for example, because they are too close to a line segment of the obstacle list or because the only path to reach them goes through a line segment of the obstacle list) are discharged. A maximum of 50 reachable candidate observation positions are generated.

- Evaluate each candidate observation position $p$ according to an evaluation function $f(p)$. The different strategies we experimentally compared use different implementations of the evaluation function $f(\cdot)$.

- Select the best position $\bar{p}$ that maximizes the function $f(\cdot), \bar{p}=\max _{p} f(p)$.

In the following, we present the exploration strategies we experimentally compared, without any attempt to provide a comprehensive discussion of their details; please refer to the original papers for more information.

\section{A. Random Strategy}

With the random strategy, the next observation position is chosen at random. This means that $f(\cdot)$ evaluates a candidate position $p$ with a random value $f(p)$. This strategy mimics exploration strategies like [12], [13] and has been selected because it constitutes a "bottom line" to which other strategies can be compared, as it is usually done in literature (for example, see [4]).

\section{B. Greedy Strategy}

The greedy strategy evaluates a candidate observation position $p$ only on the basis of its utility, namely only on the basis of the amount of new information about the environment that the robot is expected to gain from $p$. We implemented this strategy by considering a $f(\cdot)$ formed only by the first three terms of Eq. 1 (discussed below). We included this strategy in our comparison because other strategies that include cost in $f(\cdot)$ can be compared to the greedy strategy for assessing the contribution of cost to the selection of the next best observation position. Greedy strategy is usually considered when evaluating other exploration strategies [2], [4], [5].

\section{GB-L Strategy}

The Gonzáles-Baños and Latombe's exploration strategy (GB-L strategy) is presented in [19]. The strategy evaluates a candidate observation position $p$ by:

$$
f(p)=A(p) \cdot \exp (-\lambda \cdot L(p))
$$

where $A(p)$ is an estimate of the unexplored area visible from $p, L(p)$ is the length of the path connecting the current robot position and $p$, and $\lambda$ (set to $20^{-1} \mathrm{~cm}$ ) weights the new information obtainable from a position and the cost of travelling to reach the position. We calculated $A(p)$ as the difference between the area of a circle with radius $r$ centered in $p$ and the area of its intersection with the known space. $L(p)$ is calculated summing up the lengths of the line segments composing the path from the current position of the robot to $p$.

We considered this strategy because it is representative of complex exploration strategies that use ad hoc forms for $f(\cdot)$. The above formula for $f(\cdot)$ is justified by its experimental effectiveness but not theoretically.

\section{A-C-G Strategy}

In [21], we proposed an information-based exploration strategy (A-C-G strategy) that has been derived using the concept of relative entropy. The evaluation function used to compare the candidate observation positions is:

$$
\begin{gathered}
f(p)=\frac{1}{N+A} \sum_{i \in \mathcal{A} \cup \mathcal{N}} \ln \frac{\sigma_{u n c, i}}{\sigma}+N \ln \frac{\sigma}{P}+ \\
\sum_{i \in \mathcal{A}} \ln \frac{\sigma}{\sigma_{p, i}}+N \ln \frac{2 \pi \times c}{\sigma}
\end{gathered}
$$

where $N=|\mathcal{N}|$ is the expected number of new points sensed from $p, A=|\mathcal{A}|$ is the expected number of already sensed points that are sensed again from $p, \sigma_{u n c, i}$ is the standard deviation of the contribution to the measurement error due to the robot pose uncertainty, $\sigma$ is the standard deviation of the sensor accuracy, $P$ is the expected perimeter of the area to be mapped, $\sigma_{p, i}$ is the prior standard deviation of the already sensed point $i$, and $c$ is the distance between the current position of the robot and $p$. In this case, the smallest values of $f(\cdot)$ identify the best observation positions. The first term of Eq. 1 is the contribution to the entropy of the points sensed from $p$, the second term of Eq. 1 is the contribution to the entropy of the points sensed for the first time from $p$, the third term of Eq. 1 is the contribution to the entropy of the already known points that are sensed again from $p$, and the last term of Eq. 1 is the contribution to the entropy of the cost of reaching $p$. In general, the first and the fourth terms increase entropy, while the second and the third terms reduce entropy.

We calculated these quantities as follows. $\mathcal{A}$ is the set of points already perceived that lie within the circle of radius $r$ centered in $p . N$ is calculated as the difference between the number of points perceived in the last step and $A$; the implicit assumption here is that the environment is enough regular, namely that the number of sensed points at each step is roughly constant. $\sigma_{u n c, i}$ is equal to $V_{\theta} d^{2}+V_{x y}$, where $V_{\theta}$ is the expected error in the rotational position of the robot in $p, d$ is the distance between the current position of the robot and $p$, and $V_{x y}$ is the expected error in the translational position of the robot in $p$ (in our experiments $V_{\theta}=0.01$ and $\left.V_{x y}=0.001\right) . \sigma=0.01$ and $\sigma_{p, i}=2 \sigma$. Finally, $c$ is calculated summing up the lengths of the line segments composing the path from the current position of the robot to $p$.

We explicitly note that, in order to determine the next best observation position, this exploration strategy blends together 
the expected information gathered by the sensing activity (the first three terms of Eq. 1) and the distance travelled by the robot to reach the position (the last term of Eq. 1). The expected information gain is derived both from points which became visible from the observation position and from the reduction of uncertainty on the location of previously observed points.

We considered this strategy because it represent an example of complex exploration strategies that are theoretically founded in information theory.

\section{EXPERIMENTAL RESULTS}

\section{A. Setup}

We used a software simulator (also used in [2]), in which it is possible to define the environment to be explored, the number of exploring robots (1, in our case), their sensor ranges, and their initial positions. The simulator is implemented in $\mathrm{C}++$, using the LEDA libraries [23] for data structures and graphics.

The exploration strategies have been evaluated in three different environments (Fig. 1, the unit shown in the figures is $30 \mathrm{~m}$ ). The first one (office environment) is a portion of an office-like environment with three corridors. The second one (open environment) is a large open space, like a large outdoor place. The third one (obstacle environment) is a large space scattered with several obstacles.

For each environment, strategy, range for the robot sensor ( $r=10 \mathrm{~m}, r=15 \mathrm{~m}$, or $r=20 \mathrm{~m}$ ), and percentage $C$ of the free environment to be covered $(85 \%, 90 \%$, or $95 \%)$, we performed 10 trials. Since in the office environment we used only $C=95 \%$ and since we did not consider $r=$ $10 \mathrm{~m}$ in open and obstacle environments, we performed 600 trials, each one lasting from few seconds to some hours. Differences between trials are due to the initial position of the robot in the environment (randomly determined at the beginning of the trial and the same for all strategies) and to the generation of candidate observation positions (randomly generated on the free edges, as discussed in the previous section).

Two parameters have been considered to compare the performances of the exploration strategies: the number of sensing operations (steps) needed to complete the exploration and the total distance travelled by the robot during the exploration (similarly to [5], [11], [15]).

\section{B. Results}

For the office environment, the average performances (over 10 trials) of the strategies are reported in Table I. In parentheses, we report also the standard deviation for the distance metric (the standard deviation for the number of steps is almost uninformative). These results have been obtained with $C=95 \%$.

For the open and obstacle environments, the average performances (over 10 trials) of the strategies are reported in Table II and in Table III, respectively. We did not perform experiments with $r=10$ because they were too

\begin{tabular}{|c|c|c|c|c|c|c|}
\hline & \multicolumn{3}{|c|}{ \# OF STEPS } & \multicolumn{3}{c|}{ DISTANCE } \\
\hline SENSOR RANGE & 10 & 15 & 20 & 10 & 15 & 20 \\
\hline \hline random & 120.6 & 57.8 & 33.7 & $66626.7(2265.8)$ & $3473.0(1158.2)$ & $1990.4(349.3)$ \\
greedy & 116.0 & 58.5 & 37.3 & $1915.0(236.6)$ & $2390.0(874.5)$ & $2359.0(505.9)$ \\
GB-L & 127.8 & 64.8 & 40.5 & $1655.4(203.3)$ & $1121.0(122.0)$ & $866.9(74.6)$ \\
A-C-G & 119.7 & 54.6 & 35.2 & $1673.2(214.9)$ & $1132.4(147.3)$ & $909.8(110.0)$ \\
\hline
\end{tabular}

TABLE I: Office environment

\begin{tabular}{|c|c|c|c|c|c|}
\hline & & \multicolumn{2}{|c|}{ \# OF STEPS } & \multicolumn{2}{|c|}{ DISTANCE } \\
\hline & SENSOR RANGE & 15 & 20 & 15 & 20 \\
\hline \multirow{4}{*}{$C=85 \%$} & random & 164.0 & 95.4 & $9241.0(1824.9)$ & $6503.0(1733.2)$ \\
\hline & greedy & 163.5 & 91.7 & $3884.9(576.0)$ & $2900.8(357.0)$ \\
\hline & GB-L & 185.3 & 110.1 & $4150.4(436.9)$ & $3061.9(427.5)$ \\
\hline & A-C-G & 164.9 & 96.3 & $3410.3(324.9)$ & 2441.9 (180.3) \\
\hline \multirow{4}{*}{$C=90 \%$} & random & 173.9 & 101.1 & $10700.1(2299.4)$ & $6672.5(834.2)$ \\
\hline & greedy & 177.9 & 103.9 & 4315.9 (599.1) & $3404.2(522.6)$ \\
\hline & GB-L & 197.3 & 115.7 & $4332.0(417.2)$ & 3119.4 (208.5) \\
\hline & A-C-G & 182.5 & 104.5 & $3840.7(344.2)$ & $2164.7(282.2)$ \\
\hline \multirow{4}{*}{$C=95 \%$} & random & 191.3 & 107.8 & $11749.7(1462.7)$ & $7255.3(1052.7)$ \\
\hline & greedy & 194.7 & 111.9 & $4841.8(559.4)$ & $3737.3(418.4)$ \\
\hline & GB-L & 208.8 & 122.7 & $4767.8(474.2)$ & $3490.9(327.6)$ \\
\hline & A-C-G & 197.3 & 111.2 & $4312.5(284.4)$ & $3044.1(341.1)$ \\
\hline
\end{tabular}

TABLE II: Open environment

computationally expensive (several hours per trial) in such large environments.

From the tables, it can be seen that, somehow surprisingly, the small office environment is not always mapped faster than the large obstacle environment. We note also that mapping the obstacle environment is faster that mapping the open environment. This somehow counterintuitive results can be explained recalling that the termination condition of the exploration is relative to the percentage $C$ of free area of the environment to be covered. The amount of free area is smaller in the obstacle environment than in the free environment and so the exploration ends faster in the first case than in the second one. From Tables I, II, and III it can noted also that, in all environments, the larger the range of sensor, the faster the exploration; similarly the larger $C$, the longer the exploration.

It is interesting to compare the performances of the exploration strategies in the open and in the obstacle environments. Considering the number of steps, there is not very much difference between the considered strategies. This is due to the fact that the candidate observation positions are generated along the free edges for all the strategies. Any selected observation position is therefore a good view-point over the unexplored area. However, when considering the distance travelled by the robot, the random selection of the next observation position among the candidate ones shows its inefficiency. The greedy strategy seems to perform worst that GB-L and A-C-G strategies in both large environments

\begin{tabular}{|c|c|c|c|c|c|}
\hline & & \multicolumn{2}{|c|}{ \# OF STEPS } & \multicolumn{2}{|c|}{ DISTANCE } \\
\hline & SENSOR RANGE & 15 & 20 & 15 & 20 \\
\hline \multirow{4}{*}{$C=85 \%$} & random & 50.2 & 27.6 & 2671.9 (1222.0) & $1403.9(674.9)$ \\
\hline & greedy & 44.6 & 27.2 & 816.7 (175.4) & $820.4(450.4)$ \\
\hline & GB-L & 46.9 & 26.4 & $688.6(86.1)$ & $509.2(64.6)$ \\
\hline & A-C-G & 45.9 & 27.0 & $708.1(81.1)$ & $568.7(111.0)$ \\
\hline \multirow{4}{*}{$C=90 \%$} & random & 53.1 & 29.8 & $2854.6(831.7)$ & $1688.1(603.1)$ \\
\hline & greedy & 49.2 & 29.3 & $941.9(279.0)$ & 934.9 (513.6) \\
\hline & GB-L & 49.4 & 28.5 & $730.3(84.7)$ & $549.4(85.6)$ \\
\hline & A-C-G & 47.5 & 28.6 & 737.7 (102.4) & $615.9(149.0)$ \\
\hline \multirow{4}{*}{$C=95 \%$} & random & 56.5 & 31 & $2880.0(930.5)$ & 1735.6 (791.6) \\
\hline & greedy & 52.8 & 30.8 & $1027.4(408.4)$ & $985.2(495.5)$ \\
\hline & GB-L & 52.3 & 29.7 & $767.4(83.0)$ & $576.6(72.2)$ \\
\hline & A-C-G & 51 & 30.9 & 777.7 (81.7) & $692.3(194.5)$ \\
\hline
\end{tabular}

TABLE III: Obstacle environment 


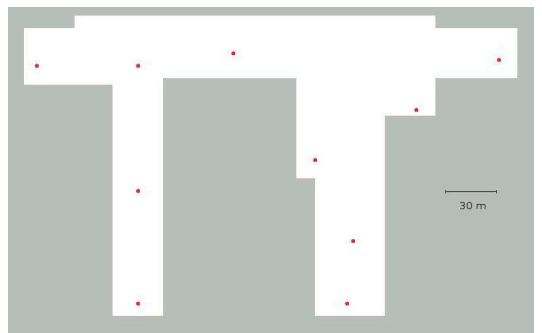

(a) office

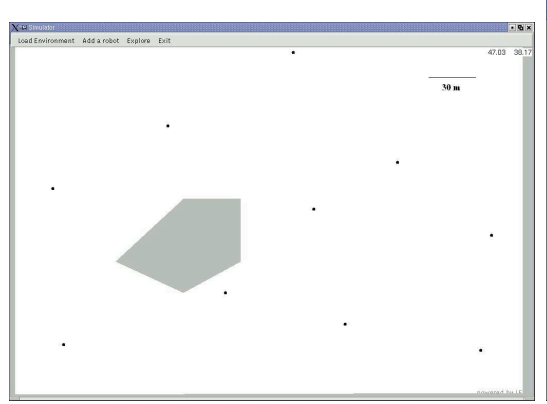

(b) open

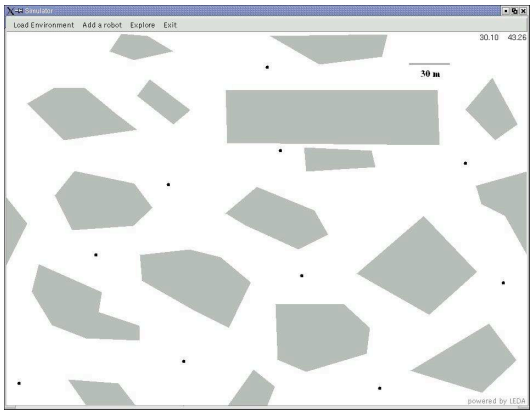

(c) obstacle

Fig. 1: The three environments (points represent different starting positions for the robot)

demonstrating that the introduction of the cost in computing $f(\cdot)$ can provide some advantages. In open environments, the A-C-G strategy seems to perform better than the GBL strategy, while the two are almost equivalent in obstacle environment (A-C-G performs slightly better in terms of number of steps, while GB-L performs better in terms of distance travelled). This leaves open the question if an ad hoc definition is preferable to a theoretically-founded definition of $f(\cdot)$.

To validate these intuitions, we analyzed the data from the open environment and the data from the obstacle environment using a one-way analysis of variance (ANOVA) [24]. We also applied a series of post hoc procedures aimed at identifying groups of strategies which performed similarly in terms of distance metric. In the open environment, the analysis of variance showed that the performance of the four strategies was significant with a probability of $99.99 \%$. The next post hoc procedures (Student-Newman-Keuls, Tukey HSD, and Scheffé) identified two groups: one consisting of the random strategy, one consisting of the other three strategies. Thus, all the strategies except the random strategy perform similarly. Also in the obstacle environment, the ANOVA showed that the four strategies performed significantly different, with a probability of $99.99 \%$. However, in this case, three post hoc procedures (Student-NewmanKeuls, Tukey HSD, and Tukey B) identified three groups: one consisting of the random strategy, one consisting of the greedy strategy, and one consisting of the GB-L and A-C$\mathrm{G}$ strategies. That is, GB-L and A-C-G strategies perform similarly (not statistically different) but their performance is significantly different from the random strategy (as in the previous case) and from the greedy strategy. In contrast, the Scheffé test confirmed what showed by the previous environment: it identified the same two groups: one for the random strategy, one for the other three strategies.

It is also interesting to analyze how the performances of the exploration strategies scale with the percentage of environment to be explored. For example, Fig. 2 shows the distance travelled by the robot when exploring the obstacle environment with $r=15 \mathrm{~m}$ according to the different strategies. The distance travelled with GB-L and A-C-G strategies slowly grows linearly with the percentage of the environment to be explored. This can be explained by the fact that the two strategies map uniformly the environment, without leaving "holes" of unexplored areas. Similar results hold also for the open environment and for different values of $r$.

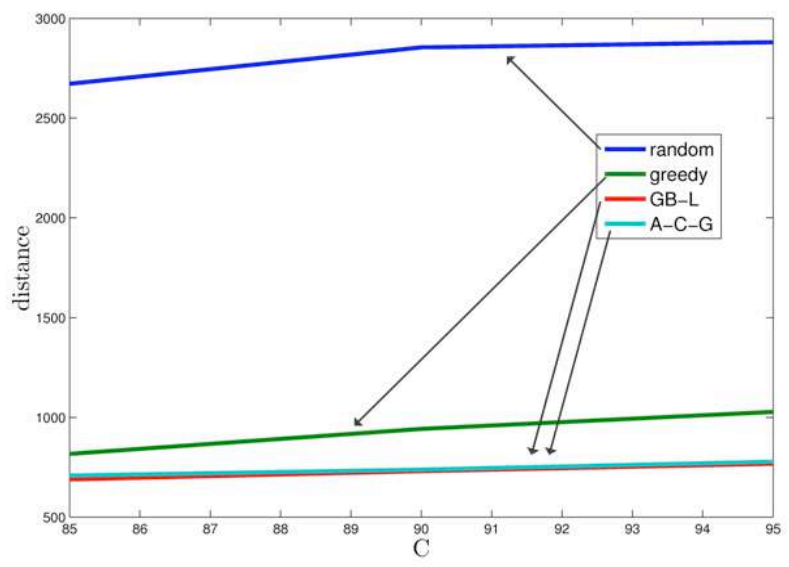

Fig. 2: Distance travelled vs. $C$

Finally, we compare the results we obtained with other experimental results reported in literature. In [5], it is shown that a greedy exploration strategy (namely, an exploration strategy that only cares about the amount of new information it expects to obtain from a view-point) has good performances when considering the number of steps but not when considering the distance travelled. With this last metric, exploration strategies that consider also the cost of reaching a view-point perform better. This result is also reflected by our experiments. Moreover, in [6], exploration strategies that try to evaluate the utility of a candidate observation position are shown to be the most effective when compared with basic exploration strategies that do not evaluate the worthiness of candidate observation positions. Also this result is confirmed by our findings. 


\section{CONCLUSIONS}

In this paper, we have experimentally compared four exploration strategies that mobile robots can employ in mapping an unknown environment. Although such comparative analysis is rather uncommon in literature on robot mapping, it is useful to evidence and quantify the strengths and weaknesses of different approaches. For example, with our analysis we confirmed that exploration strategies that balance utility and cost (like GB-L and A-C-G strategies) tend to be more efficient than those that use only utility (like greedy strategy). However, some issues, like the comparison between ad hoc and theoretically-based strategies, are still open.

The results presented in this paper constitutes only a step toward a comprehensive evaluation of different exploration strategies. For example, more environments and more strategies need to be tested to assess stronger conclusions that can influence the future development of exploration strategies. Moreover, some important aspects, including the effects of uncertainty and the extension to outdoor environments, have not been fully considered in this paper and deserve more attention.

\section{ACKNOWLEDGMENTS}

The author gratefully thanks Andrea Quarta for contribution is producing the experimental data and Pier Luca Lanzi for contribution in performing statistical analysis.

\section{REFERENCES}

[1] S. Thrun, "Robotic mapping: A survey," in Exploring Artificial Intelligence in the New Millenium. Morgan Kaufmann, 2002, pp. 1-35.

[2] F. Amigoni and A. Gallo, "A multi-objective exploration strategy for mobile robots," in Proceedings of the IEEE International Conference on Robotics and Automation, 2005, pp. 3861-3866.

[3] W. Burgard, M. Moors, C. Stachniss, and F. Schneider, "Coordinated multi-robot exploration," IEEE Transactions on Robotics, vol. 21, no. 3, pp. 376- 386, 2005.

[4] R. Sim and N. Roy, "Global a-optimal robot exploration in SLAM," in Proceedings of the IEEE International Conference on Robotics and Automation, 2005, pp. 661-666.

[5] C. Stachniss and W. Burgard, "Exploring unknown environments with mobile robots using coverage maps," in Proceedings of the 18th Joint Conference on Artificial Intelligence, 2003, pp. 1127-1134.

[6] D. Lee and M. Recce, "Quantitative evaluation of the exploration strategies of a mobile robot," International Journal of Robotics Research, vol. 16, no. 4, pp. 413-447, 1997.

[7] S. Thrun, W. Burgard, and D. Fox, "A real-time algorithm for mobile robot mapping with applications to multi-robot and 3D mapping," in Proceedings of the IEEE International Conference on Robotics and Automation, 2000, pp. 321-328.

[8] F. Amigoni, S. Gasparini, and M. Gini, "Building segment-based maps without pose information," Proceedings of the IEEE, vol. 94, no. 7, pp. 1340-1359, 2006.

[9] F. Lu and E. Milios, "Robot pose estimation in unknown environments by matching 2D range scans," Journal of Intelligent and Robotic Systems, vol. 18, no. 3, pp. 249-275, 1997.

[10] R. Sim and G. Dudek, "Effective exploration strategies for the construction of visual maps," in Proceedings of the IEEE/RSJ International Conference on Intelligent Robots and Systems, 2003, pp. 32243231.

[11] R. Sim, G. Dudek, and N. Roy, "Online control policy optimization for minimizing map uncertainty during exploration," in Proceedings of the IEEE International Conference on Robotics and Automation, 2004, pp. 1758-1762.
[12] L. Freda and G. Oriolo, "Frontier-based probabilistic strategies for sensor-based exploration," in Proceedings of the IEEE International Conference on Robotics and Automation, 2005, pp. 3881-3887.

[13] D. Schmidt, T. Luksch, J. Wettach, and K. Berns, "Autonomous behavior-based exploration of office environments," in Proceedings of the International Conference on Informatics in Control, Automation and Robotics, 2006, pp. 235-240.

[14] C. Tovey and S. Koenig, "Improved analysis of greedy mapping," in Proceedings of the IEEE/RSJ International Conference on Intelligent Robots and Systems, 2003.

[15] B. Tovar, L. Muñoz-Gómez, R. Murrieta-Cid, M. Alencastre-Miranda, R. Monroy, and S. Hutchinson, "Planning exploration strategies for simultaneous localization and mapping," Robotics and Autonomous Systems, vol. 54, pp. 314-331, 2006.

[16] B. Yamauchi, "A frontier-based approach for autonomous exploration," in Proceedings of the IEEE International Symposium on Computational Intelligence in Robotics and Automation, 1997, pp. 146-151.

[17] P. Newman, M. Bosse, and J. Leonard, "Autonomous feature-based exploration," in Proceedings of the IEEE International Conference on Robotics and Automation, 2003, pp. 1234-1240.

[18] T. Edlinger and E. von Puttkamer, "Exploration of an indoorenvironment by an autonomous mobile robot," in Proceedings of the IEEE/RSJ International Conference on Intelligent Robots and Systems, 1994 , pp. $1278-1284$.

[19] H. H. Gonzáles-Baños and J.-C. Latombe, "Navigation strategies for exploring indoor environments," International Journal of Robotics Research, vol. 21, no. 10-11, pp. 829-848, 2002.

[20] A. Makarenko, S. Williams, F. Bourgault, and H. Durrant-Whyte, "An experiment in integrated exploration," in Proceedings of the IEEE/RSJ International Conference on Intelligent Robots and Systems, 2002, pp. 534-539.

[21] F. Amigoni, V. Caglioti, and U. Galtarossa, "A mobile robot mapping system with an information-based exploration strategy," in Proceedings of the International Conference on Informatics in Control, Automation and Robotics, 2004, pp. 71-78.

[22] R. Rocha, J. Dias, and A. Carvalho, "Cooperative multi-robot systems: A study of vision-based 3-D mapping using information theory," Robotics and Autonomous Systems, vol. 53, pp. 282-311, 2005.

[23] LEDA Library, http://www.algorithmic-solutions.com/enleda.htm.

[24] S. A. Glantz and B. K. Slinker, Primer of Applied Regression and Analysis of Variance. McGraw-Hill/Appleton \& Lange, 2000. 